\title{
Polymerase chain reaction for Cryptosporidium in the immunocompromised host
}

\author{
Luis Ignacio Gonzalez-Granado
}

Received: 3 September 2009 / Accepted: 16 September 2009/Published online: 10 November 2009

(C) IPNA 2009

Sirs,

I read with great interest the article by Bandin et al. entitled "Cryptosporidiosis in paediatric renal transplantation" [1]. I am grateful for their contribution to the knowledge of Cryptosporidium's impact on pediatric kidney transplantation. However, I would like to make some comments.

First, the diagnosis in the immunocompromised host is more difficult than in the immunocompetent one. Specific Ziehl-Nielsen staining techniques have low sensitivity, especially compared with polymerase chain reaction [2]. So many cases in kidney transplantation can be missed; therefore, the real prevalence in this cohort could have been higher. This should alert everyone to rule out with appropriate diagnostic testing the presence of Cryptosporidium parvum in the stools of pediatric patients who receive kidney transplantation.

Second, no drug has proven efficacy in the severely immunocompromised host against C. parvum. Unlikely positive results can be achieved with nitazoxanide [3], paromomycin [4] or the combination of paromomycin and azithromycin [5]. The best efficacy is obtained by lowering immunosuppression [6].

\section{References}

1. Bandin F, Kwon T, Linas MD, Guigonis V, Valentin A, Cassaing S, Carol A, Garnier A, Baudouin V, Decramer S (2009) Cryptosporidiosis in paediatric renal transplantation. Pediatr Nephrol. doi:10.1007/s00467-009-1274-y

2. McLauchlin J, Amar CF, Pedraza-Díaz S, Mieli-Vergani G, Hadzic N, Davies EG (2003) Polymerase chain reaction-based diagnosis of infection with Cryptosporidium in children with primary immunodeficiencies. Pediatr Infect Dis J 22:329-335

3. Rossignol JF (2006) Nitazoxanide in the treatment of acquired immune deficiency syndrome-related cryptosporidiosis results of the United States compassionate use program in 365 patients. Aliment Pharmacol Ther 24:887-894

4. Maggi P, Larocca AM, Ladisa N, Carbonara S, Brandonisio O, Angarano G, Pastore G (2001) Opportunistic parasitic infections of the intestinal tract in the era of highly active antiretroviral therapy: is the CD4(+) count so important? Clin Infect Dis 33:1609-1611

5. Smith NH, Cron S, Valdez LM, Chappell CL, White AC Jr (1998) Combination drug therapy for cryptosporidiosis in AIDS. J Infect Dis 178:900-903

6. Abubakar I, Aliyu SH, Arumugam C, Hunter PR, Usman NK (2007) Prevention and treatment of cryptosporidiosis in immunocompromised patients. Cochrane Database Syst Rev (1): CD004932
L. I. Gonzalez-Granado $(\bowtie)$

Immunodeficiencies Unit, Hospital 12 octubre,

Av Andalucía Km 5,400,

28041 Madrid, Spain

e-mail: nachgonzalez@gmail.com 